\title{
EXACT DECAY RATE OF A NONLINEAR ELLIPTIC EQUATION RELATED TO THE YAMABE FLOW
}

\author{
SHU-YU HSU \\ (Communicated by Walter Craig)
}

\begin{abstract}
Let $0<m<\frac{n-2}{n}, n \geq 3, \alpha=\frac{2 \beta+\rho}{1-m}$ and $\beta>\frac{m \rho}{n-2-m n}$ for some constant $\rho>0$. Suppose $v$ is a radially symmetric solution of $\frac{n-1}{m} \Delta v^{m}+\alpha v+\beta x \cdot \nabla v=0, v>0$, in $\mathbb{R}^{n}$. When $m=\frac{n-2}{n+2}$, the metric $g=v^{\frac{4}{n+2}} d x^{2}$ corresponds to a locally conformally flat Yamabe shrinking gradient soliton with positive sectional curvature. We prove that the solution $v$ of the above nonlinear elliptic equation has the exact decay rate $\lim _{r \rightarrow \infty} r^{2} v(r)^{1-m}=\frac{2(n-1)(n(1-m)-2)}{(1-m)(\alpha(1-m)-2 \beta)}$.
\end{abstract}

\section{INTRODUCTION}

Recently, there has been a lot of study of the equation

$$
\frac{n-1}{m} \Delta v^{m}+\alpha v+\beta x \cdot \nabla v=0, \quad v>0, \quad \text { in } \mathbb{R}^{n}
$$

where

$$
0<m<\frac{n-2}{n}, \quad n \geq 3,
$$

and

$$
\alpha=\frac{2 \beta+\rho}{1-m}
$$

for some constant $\rho \in \mathbb{R}$ by P. Daskalopoulos and N. Sesum [DS2]; S.Y. Hsu [H1], [H2]; M.A. Peletier and H. Zhang [PZ]; and J.L. Vázquez [V1]. In the paper [DS2] P. Daskalopoulos and N. Sesum (cf. [CSZ, CMM]) proved the important result that any locally conformally flat non-compact gradient Yamabe soliton $g$ with positive sectional curvature on an $n$-dimensional manifold, $n \geq 3$, must be radially symmetric and have the form $g=v^{\frac{4}{n+2}} d x^{2}$, where $d x^{2}$ is the Euclidean metric on $\mathbb{R}^{n}$ and $v$ is a radially symmetric solution of (1.1) with $m=\frac{n-2}{n+2}$, and $\alpha, \beta$ satisfy (1.3) for some constant $\rho>0, \rho=0$ or $\rho<0$, depending on whether $g$ is a shrinking, steady, or expanding Yamabe soliton.

On the other hand, as observed by B.H. Gilding, M.A. Peletier and H. Zhang GP, [PZ, and others ( DS1, DS2, V1], V2 ), (1.1) also arises in the study of the self-similar solutions of the degenerate diffusion equation

$$
u_{t}=\frac{n-1}{m} \Delta u^{m} \quad \text { in } \mathbb{R}^{n} \times(0, T) .
$$

Received by the editors November 14, 2012 and, in revised form, January 12, 2013.

2010 Mathematics Subject Classification. Primary 35J70, 35B40; Secondary 58J37, 58J05.

Key words and phrases. Nonlinear elliptic equation, Yamabe soliton, exact decay rate. 
For example (cf. [H1, V1]) if $v$ is a radially symmetric solution of (1.1) with

$$
\alpha=\frac{2 \beta+1}{1-m}>0
$$

then for any $T>0$ the function

$$
u(x, t)=(T-t)^{\alpha} v\left(x(T-t)^{\beta}\right)
$$

is a solution of (1.4) in $\mathbb{R}^{n} \times(-\infty, T)$. We refer the reader to the book V1] and the paper [H1 for the relation between solutions of (1.1) and the other self-similar solutions of (1.4) for the other parameter ranges of $\alpha, \beta$.

Note that when $v$ is a radially symmetric solution of (1.1), then $v$ satisfies

$$
\frac{n-1}{m}\left(\left(v^{m}\right)^{\prime \prime}+\frac{n-1}{r}\left(v^{m}\right)^{\prime}\right)+\alpha v+\beta r v^{\prime}=0, \quad v>0, \quad \text { in }(0, \infty)
$$

and

$$
\left\{\begin{array}{l}
v(0)=\eta, \\
v^{\prime}(0)=0,
\end{array}\right.
$$

for some constant $\eta>0$. Existence of solutions of (1.6), (1.7), for the case $n \geq 3$, $0<m \leq(n-2) / n, \beta>0$ and $\alpha \leq \beta(n-2) / m$ is proved by S.Y. Hsu in [H1. On the other hand, by the result of [PZ] and Theorem 7.4 of [V1] if (1.2) holds, then there exists a constant $\bar{\beta}$ with $\bar{\beta}=0$ when $m=\frac{n-2}{n+2}$ such that for any $\alpha=\frac{2 \beta+1}{1-m}$ and $\beta>\bar{\beta}$, there exists a unique solution of (1.6), (1.7). Moreover, if $0<\alpha=\frac{2 \beta+1}{1-m}$ and $\beta<\bar{\beta}$, then (1.6), (1.7) have no global solution.

Since the asymptotic behavior of solutions of (1.4) is usually similar to the behavior of the radially symmetric self-similar solutions of (1.4), in order to understand the asymptotic behavior of solutions of (1.4) and the asymptotic behavior of locally conformally flat non-compact gradient Yamabe solitons, it is important to study the asymptotic behavior of the solutions of (1.6), (1.7).

Exact decay rate of the solutions of (1.6), (1.7) for the case

$$
\alpha=\frac{2 \beta}{1-m}>0
$$

and the case

$$
\frac{2 \beta}{1-m}>\max (\alpha, 0)
$$

with $m, n$ satisfying (1.2), was obtained by S.Y. Hsu in [H1. When (1.2) and (1.3) hold for some constant $\rho>0$, although it is known (DS2, V1) that solution $v$ of (1.6), (1.7) satisfies $v(r)=O\left(r^{-\frac{2}{1-m}}\right)$ as $r \rightarrow \infty$, nothing is known about the exact decay rate of $v$. In [H2] S.Y. Hsu proved, by using estimates for the scalar curvature of the metric $g=v^{\frac{4}{n+2}} d x^{2}$ where $v$ is a radially symmetric solution of (1.1), that when $m=\frac{n-2}{n+2}, \beta>\frac{\rho}{n-2}>0$,

$$
\lim _{r \rightarrow \infty} r^{2} v(r)=\frac{(n-1)(n-2)}{\rho} .
$$

In this paper we will extend the above result and prove the exact decay rate of radially symmetric solution $v$ of (1.1) when (1.2) and (1.3) hold for some constant $\rho>0$. More precisely we will prove the following theorem. 
Theorem 1.1. Let $\eta>0, \rho>0, m, n, \alpha, \beta$, satisfy (1.2), (1.3), and

$$
\beta>\frac{m \rho}{n-2-m n} \text {. }
$$

Suppose $v$ is a solution of (1.6), (1.7). Then

$$
\lim _{r \rightarrow \infty} r^{2} v(r)^{1-m}=\frac{2(n-1)(n(1-m)-2)}{(1-m)(\alpha(1-m)-2 \beta)} .
$$

Remark 1.2. The function

$$
v_{0}(x)=\left(\frac{2(n-1)(n(1-m)-2)}{(1-m)(\alpha(1-m)-2 \beta)|x|^{2}}\right)^{\frac{1}{1-m}}
$$

is a singular solution of (1.1) in $\mathbb{R}^{n} \backslash\{0\}$. If $v$ is a solution of (1.1), then for any $\lambda>0$ the function

$$
v_{\lambda}(x)=\lambda^{\frac{2}{1-m}} v(\lambda x)
$$

is also a solution of (1.1).

Corollary 1.3. Let $\rho, m, n, \alpha, \beta$ satisfy (1.2), (1.3), (1.9). Suppose $v$ is a radially symmetric solution of (1.1), and $v_{0}, v_{\lambda}$ are given by (1.11) and (1.12), respectively. Then $v_{\lambda}(x)$ converges uniformly on $\mathbb{R}^{n} \backslash B_{R}(0)$ to $v_{0}(x)$ for any $R>0$ as $\lambda \rightarrow \infty$.

Corollary 1.4 (cf. [H2]). The metric $g_{i j}=v^{\frac{4}{n+2}} d x^{2}, n \geq 3$, of a locally conformally flat non-compact gradient shrinking Yamabe soliton where $v$ is radially symmetric and satisfies (1.1) with $m=\frac{n-2}{n+2}$, and $\beta>\frac{\rho}{2}>0, \alpha$, satisfying (1.3) has the exact decay rate (1.8).

Since the scalar curvature of the metric $g_{i j}=v^{\frac{4}{n+2}} d x^{2}, n \geq 3$, where $v$ is a radially symmetric solution of (1.1) with $m=\frac{n-2}{n+2}$ is given by ([DS2, [H2])

$$
R(r)=(1-m)\left(\alpha+\beta \frac{r v^{\prime}(r)}{v(r)}\right),
$$

by Corollary 1.4 and an argument similar to the proof of Lemma 3.4 and Theorem 1.3 of [H2], we obtain the following extensions of Theorem 1.2 and Theorem 1.3 of $[\mathrm{H} 2]$.

Theorem 1.5. Let $m=\frac{n-2}{n+2}, n \geq 3, \beta>\frac{\rho}{2}>0$, $\alpha$, satisfy (1.3). Let $v$ be $a$ radially symmetric solution of (1.1). Then

$$
\lim _{r \rightarrow \infty} \frac{r v^{\prime}(r)}{v(r)}=-\frac{2}{1-m}
$$

and the scalar curvature $R(r)$ of the metric $g_{i j}=v^{\frac{4}{n+2}} d x^{2}$ satisfies

$$
\lim _{r \rightarrow \infty} R(r)=\rho .
$$

If $K_{0}$ and $K_{1}$ are the sectional curvatures of the 2-planes perpendicular to and tangent to the spheres $\{x\} \times S^{n-1}$, respectively, then

$$
\lim _{r \rightarrow \infty} K_{0}(r)=0
$$

and

$$
\lim _{r \rightarrow \infty} K_{1}(r)=\frac{\rho}{(n-1)(n-2)}
$$


Corollary 1.6. Let $\eta>0, \rho>0, m, n, \alpha, \beta$ satisfy (1.2), (1.3), and (1.9). Suppose $v$ is a solution of (1.6), (1.7). Then (1.13) holds.

The plan of the paper is as follows. We will prove the boundedness of the function

$$
w(r)=r^{2} v(r)^{1-m}
$$

where $v$ is the solution of (1.1) in section two. We will also find the lower bound of $w$ in section two. In section three we will prove Theorem 1.1 and Corollary 1.3. We will assume that (1.2), (1.3) hold for some constant $\rho>0$ and let $v$ be a radially symmetric solution of (1.1) or equivalently the solution of (1.6), (1.7), for some $\eta>0$, and

$$
w_{\infty}=\frac{2(n-1)(n(1-m)-2)}{(1-m)(\alpha(1-m)-2 \beta)}
$$

for the rest of the paper. Note that when $\alpha=n \beta$ and $\alpha=\frac{2 \beta+1}{1-m}$, the solution of (1.1) is given explicitly by (cf. [DS2])

$$
v_{\lambda}(x)=\left(\frac{2(n-1)(n-2-n m)}{(1-m)\left(\lambda^{2}+|x|^{2}\right)}\right)^{\frac{1}{1-m}}, \quad \lambda>0,
$$

which satisfies (1.10).

\section{2. $L^{\infty}$ estimate of $w$}

Lemma 2.1. Let $\rho>0, m, n, \alpha, \beta$ satisfy (1.2) and (1.3) and let $v$ be a radially symmetric solution of (1.1). Let $w$ be given by (1.14). Suppose there exists a constant $C_{1}>0$ such that

$$
w(r) \leq C_{1} \quad \forall r \geq 1 .
$$

Then any sequence $\left\{w\left(r_{i}\right)\right\}_{i=1}^{\infty}, r_{i} \rightarrow \infty$ as $i \rightarrow \infty$, has a subsequence $\left\{w\left(r_{i}^{\prime}\right)\right\}_{i=1}^{\infty}$ such that

$$
\lim _{r \rightarrow \infty} w\left(r_{i}^{\prime}\right)=\left\{\begin{array}{llll}
0 & \text { or } & w_{\infty} & \text { if } v \notin L^{1}\left(\mathbb{R}^{n}\right), \\
0 & \text { or } & w_{1} & \text { if } v \in L^{1}\left(\mathbb{R}^{n}\right) \\
0 & & & \text { if } v \in L^{1}\left(\mathbb{R}^{n}\right) \quad \text { and } \beta>0
\end{array}\right.
$$

where

$$
w_{1}=\frac{2(n-1)}{(1-m) \beta} \quad \text { if } \beta>0 .
$$

Proof. Let $\left\{r_{i}\right\}_{i=1}^{\infty}$ be a sequence such that $r_{i} \rightarrow \infty$ as $i \rightarrow \infty$. By (2.1) the sequence $\left\{w\left(r_{i}\right)\right\}_{i=1}^{\infty}$ has a subsequence that we may assume, without loss of generality, to be the sequence itself that converges to some constant $a \in\left[0, C_{1}\right]$ as $i \rightarrow \infty$. Integrating (1.6) over $(0, r)$ and simplifying,

$$
-\frac{n-1}{m}\left(v^{m}\right)^{\prime}(r)=\beta r v(r)+\frac{\alpha-n \beta}{r^{n-1}} \int_{0}^{r} z^{n-1} v(z) d z \quad \forall r>0 .
$$

Integrating (2.4) over $(r, \infty)$, by (2.1) we get

$$
\frac{n-1}{m} v(r)^{m}=\beta \int_{r}^{\infty} s v(s) d s+\int_{r}^{\infty} \frac{\alpha-n \beta}{s^{n-1}}\left(\int_{0}^{s} z^{n-1} v(z) d z\right) d s \quad \forall r>0 .
$$


Let $b=a^{\frac{1}{1-m}}=\lim _{i \rightarrow \infty} r_{i}^{\frac{2}{1-m}} v\left(r_{i}\right)$. Then by (2.1), (2.5), and the l'Hospital rule,

$$
\begin{aligned}
\frac{(n-1)}{m} b^{m} & =\frac{(n-1)}{m} \lim _{i \rightarrow \infty}\left(r_{i}^{\frac{2}{1-m}} v(r)\right)^{m} \\
& =\beta \lim _{i \rightarrow \infty} \frac{\int_{r_{i}}^{\infty} s v(s) d s}{r_{i}^{-\frac{2 m}{1-m}}}+\lim _{i \rightarrow \infty} \frac{\int_{r_{i}}^{\infty} \frac{\alpha-n \beta}{s^{n-1}}\left(\int_{0}^{s} z^{n-1} v(z) d z\right) d s}{r_{i}^{-\frac{2 m}{1-m}}} \\
& =\frac{(1-m)}{2 m}\left(\beta \lim _{i \rightarrow \infty} \frac{r_{i} v\left(r_{i}\right)}{r_{i}^{-\frac{2 m}{1-m}-1}}+(\alpha-n \beta) \lim _{i \rightarrow \infty} \frac{\frac{1}{r_{i}^{n-1}} \int_{0}^{r_{i}} z^{n-1} v(z) d z}{r_{i}^{-\frac{2 m}{1-m}-1}}\right) \\
& =\frac{(1-m)}{2 m}\left(\beta b+(\alpha-n \beta) \lim _{i \rightarrow \infty} \frac{\int_{0}^{r_{i}} z^{n-1} v(z) d z}{r_{i}^{n-\frac{2}{1-m}}}\right) .
\end{aligned}
$$

We now divide the proof into two cases.

Case 1: $v \notin L^{1}\left(\mathbb{R}^{n}\right)$. By (2.6) and the l'Hospital rule,

$$
\begin{aligned}
\frac{(n-1)}{m} b^{m} & =\frac{(1-m)}{2 m}\left(\beta b+\frac{\alpha-n \beta}{n-\frac{2}{1-m}} \cdot \lim _{i \rightarrow \infty} \frac{r_{i}^{n-1} v\left(r_{i}\right)}{\left.r_{i}^{n-\frac{2}{1-m}-1}\right)}\right. \\
& =\frac{(1-m)}{2 m}\left(\beta b+\frac{\alpha-n \beta}{n-\frac{2}{1-m}} b\right) \\
= & \frac{(1-m)[\alpha(1-m)-2 \beta]}{2 m[n(1-m)-2]} b \\
\Rightarrow \quad a=b=0 \quad \text { or } \quad & a=b^{1-m}=w_{\infty} .
\end{aligned}
$$

Case 2: $v \in L^{1}\left(\mathbb{R}^{n}\right)$. By (2.6),

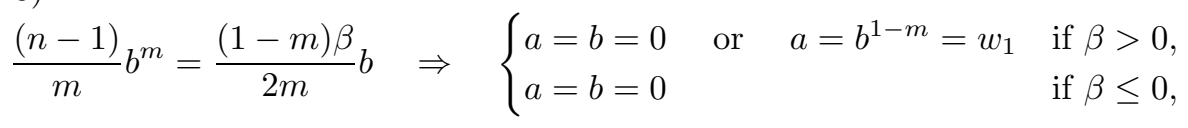

By (2.7) and (2.8) the lemma follows.

Remark 2.2. When $\beta>0, w_{1}>w_{\infty}$ if and only if $\alpha>n \beta$.

Corollary 2.3. Suppose there exist constants $C_{1}>C_{2}>0$ such that

$$
C_{2} \leq w(r) \leq C_{1} \quad \forall r \geq 1
$$

Then (1.10) holds.

Lemma 2.4. Let $\eta>0, \rho>0, \beta>0, m, n, \alpha \leq n \beta$ satisfy (1.2) and (1.3). Then

$$
v(r) \geq\left(\eta^{m-1}+\frac{(1-m) \beta}{2(n-1)} r^{2}\right)^{-\frac{1}{1-m}} \quad \forall r \geq 0 .
$$

Hence, there exists a constant $C_{2}>0$ such that

$$
w(r) \geq C_{2} \quad \forall r \geq 1
$$


Proof. (2.9) is proved on page 22 of [DS2]. For the sake of completeness, we will give a simple different proof here. By (2.4),

$$
\begin{array}{rlrl}
-\frac{n-1}{m}\left(v^{m}\right)^{\prime}(r) & \leq \beta r v(r) \quad \forall r>0 \\
\Rightarrow \quad-(n-1) v^{m-2} v^{\prime}(r) & \leq \beta r \quad \forall r>0 \\
\Rightarrow \quad & \quad \frac{n-1}{1-m}\left(v(r)^{m-1}-\eta^{m-1}\right) & \leq \frac{\beta}{2} r^{2} \quad \forall r>0
\end{array}
$$

and (2.9) follows. By (2.9), we get (2.10) and the lemma follows.

We now recall a result of $[\mathrm{H} 2]$.

Lemma 2.5 (cf. Lemma 2.3 of [H2]). Let $\eta>0, \rho>0, m, n, \alpha \geq n \beta>0$ satisfy (1.2) and (1.3). Then there exists a constant $C_{1}>0$ such that (2.1) holds.

Proof. This result is proved in [H2]. For the sake of completeness, we will repeat the proof here. By (2.4) $v^{\prime}(r)<0$ for all $r>0$. Then by (2.4),

$$
\begin{gathered}
\frac{n-1}{m} r^{n-1}\left(v^{m}\right)^{\prime}(r) \leq-\beta r^{n} v(r)-(\alpha-n \beta) \int_{0}^{r} z^{n-1} v(r) d z \\
=-\frac{\alpha}{n} r^{n} v(r) \quad \forall r>0 \\
\Rightarrow \quad v^{m-2}(r) v^{\prime}(r) \leq-\frac{\alpha}{n(n-1)} r \quad \forall r>0 \\
\Rightarrow \quad v(r) \leq\left(\eta^{m-1}+\frac{\alpha(1-m)}{2 n(n-1)} r^{2}\right)^{-\frac{1}{1-m}} \leq\left(\frac{2 n(n-1)}{\alpha(1-m)} r^{-2}\right)^{\frac{1}{1-m}} \quad \forall r>0 .
\end{gathered}
$$

Hence, (2.1) holds with $C_{1}=\frac{2 n(n-1)}{\alpha(1-m)}$ and the lemma follows.

Lemma 2.6. Let $\eta>0, \rho>0, m, n, 0<\alpha \leq n \beta$ satisfy (1.2) and (1.3). Then there exists a constant $C_{1}>0$ such that (2.1) holds.

Proof. Let $A=\left\{r \in[1, \infty): w^{\prime}(r) \geq 0\right\}$. We now divide the proof into two cases.

Case 1: $A \cap\left[R_{0}, \infty\right) \neq \phi \quad \forall R_{0}>1$. We will use a modification of the proof of Lemma 3.2 of $\left[\mathrm{H} 2\right.$ to prove this case. By Lemma 2.4 there exists a constant $C_{2}>0$ such that (2.10) holds. Hence, by (2.10),

$$
\begin{aligned}
r^{n} v(r) & =r^{n-\frac{2}{1-m}} w(r)^{\frac{1}{1-m}} \geq C_{2} r^{n-\frac{2}{1-m}} \quad \forall r \geq 1 \\
\Rightarrow \quad r^{n} v(r) & \rightarrow \infty \quad \text { as } r \rightarrow \infty .
\end{aligned}
$$

We now claim that

$$
\limsup _{\substack{r \in A \\ r \rightarrow \infty}} \frac{\int_{0}^{r} z^{n-1} v(z) d z}{r^{n} v(r)} \leq \frac{1-m}{n(1-m)-2} .
$$

We divide the proof of the above claim into two cases.

Case (1a): $\int_{0}^{\infty} z^{n-1} v(z) d z<\infty$. By (2.11) we get (2.12).

Case (1b): $\int_{0}^{\infty} z^{n-1} v(z) d z=\infty$. Since

$$
\begin{aligned}
\frac{d}{d r}\left(r^{n} v(r)\right) & =\left(n-\frac{2}{1-m}\right) r^{n-1} v(r)+\frac{1}{1-m} r^{n-\frac{2}{1-m}} w^{\frac{m}{1-m}}(r) w^{\prime}(r) \\
& \geq\left(n-\frac{2}{1-m}\right) r^{n-1} v(r) \quad \forall r \in A,
\end{aligned}
$$


by (2.11) and the l'Hospital rule,

$$
\begin{aligned}
& \limsup _{\substack{r \in A \\
r \rightarrow \infty}} \frac{\int_{0}^{r} z^{n-1} v(z) d z}{r^{n} v(r)} \\
& \quad=\limsup _{\substack{r \in A \\
r \rightarrow \infty}} \frac{r^{n-1} v(r)}{\left(n-\frac{2}{1-m}\right) r^{n-1} v(r)+\frac{1}{1-m} r^{n-\frac{2}{1-m}} w^{\frac{m}{1-m}}(r) w^{\prime}(r)} \\
& \quad \leq\left(n-\frac{2}{1-m}\right)^{-1}
\end{aligned}
$$

and (2.12) follows. Let $0<\delta<\frac{\rho}{n(1-m)-2}$. By (2.12) there exists a constant $R_{1}>1$ such that

$$
\frac{\int_{0}^{r} z^{n-1} v(z) d z}{r^{n} v(r)}<\frac{(1-m)}{n(1-m)-2}+\frac{\delta}{1+n \beta-\alpha} \quad \forall r \geq R_{1}, r \in A,
$$

$$
\Rightarrow \quad \int_{0}^{r} z^{n-1} v(z) d z \leq\left(\frac{(1-m)}{n(1-m)-2}+\frac{\delta}{1+n \beta-\alpha}\right) r^{n} v(r) \quad \forall r \geq R_{1}, r \in A .
$$

By (2.4) and (2.13),

$$
\begin{aligned}
\frac{n-1}{m} r^{n-1}\left(v^{m}\right)^{\prime}(r) & \leq-\beta r^{n} v(r)+\left(\frac{(n \beta-\alpha)(1-m)}{n(1-m)-2}+\delta\right) r^{n} v(r) \\
& \leq-\left(\frac{\rho}{n(1-m)-2}-\delta\right) r^{n} v(r) \quad \forall r \geq R_{1}, r \in A, \\
\Rightarrow \quad(n-1) v^{m-2} v^{\prime}(r) & \leq-\left(\frac{\rho}{n(1-m)-2}-\delta\right) r \quad \forall r \geq R_{1}, r \in A .
\end{aligned}
$$

Hence, there exists a constant $C_{3}>0$ such that

$$
\begin{aligned}
& \frac{r v^{\prime}(r)}{v(r)} \leq-C_{3} r^{2} v(r)^{1-m}=-C_{3} w(r) \quad \forall r \geq R_{1}, r \in A, \\
\Rightarrow \quad & 0 \leq w^{\prime}(r)=\frac{2 w(r)}{r}\left(1+\frac{1-m}{2} \cdot \frac{r v^{\prime}(r)}{v(r)}\right) \\
& \leq \frac{2 w(r)}{r}\left(1-\frac{(1-m) C_{3}}{2} w(r)\right) \quad \forall r \geq R_{1}, r \in A, \\
\Rightarrow & w(r) \leq \frac{2}{(1-m) C_{3}} \quad \forall r \geq R_{1}, r \in A .
\end{aligned}
$$

Let $r_{1} \in A \cap\left[R_{1}, \infty\right)$. Then for any $r^{\prime} \in\left(r_{1}, \infty\right) \backslash A$, there exists $r_{2} \in A \cap\left[r_{1}, \infty\right)$ such that

$$
\begin{aligned}
& w^{\prime}(r)<0 \quad \forall r_{2}<r \leq r^{\prime} \quad \text { and } \quad w^{\prime}\left(r_{2}\right)=0 \\
\Rightarrow & w\left(r^{\prime}\right) \leq w\left(r_{2}\right) \leq \frac{2}{(1-m) C_{3}} \quad \forall r^{\prime}>r_{1}, r^{\prime} \notin A \quad(\text { by (2.14) }) .
\end{aligned}
$$

By (2.14) and (2.15),

$$
w(r) \leq \frac{2}{(1-m) C_{3}} \quad \forall r \geq r_{1}
$$

and (2.1) holds with $C_{1}=\max \left(\frac{2}{(1-m) C_{3}}, \max _{1 \leq r \leq r_{1}} w(r)\right)$. 
Case 2: There exists a constant $R_{0}>1$ such that $A \cap\left[R_{0}, \infty\right)=\phi$. Then $w^{\prime}(r)<0$ for all $r \geq R_{0}$. Hence, (2.1) holds with $C_{1}=\max _{1 \leq r \leq R_{0}} w(r)$ and the lemma follows.

\section{Proof of Theorem 1.1}

We first recall a result of [H1]:

Lemma 3.1 (cf. Lemma 2.1 of [H1]). Let $\eta>0, m, n, \alpha>0, \beta \neq 0$ satisfy (1.2) and

$$
\frac{m \alpha}{\beta} \leq n-2 .
$$

Let $v$ be the solution of (1.6), (1.7). Then

$$
v(r)+\frac{\beta}{\alpha} r v^{\prime}(r)>0 \quad \forall r \geq 0
$$

and

$$
v^{\prime}(r)<0 \quad \forall r>0 .
$$

Lemma 3.2. Let $\rho>0, m, n, \alpha>n \beta$ satisfy (1.2), (1.3) and (1.9). Then

$$
\lim _{r \rightarrow \infty} r^{n-2} v^{m}(r)=\infty \text {. }
$$

Proof. Suppose (3.3) does not hold. Then there exists a sequence $\left\{r_{i}\right\}_{i=1}^{\infty}, r_{i} \rightarrow \infty$ as $i \rightarrow \infty$, such that $r_{i}^{n-2} v^{m}\left(r_{i}\right) \rightarrow a_{1}$ as $i \rightarrow \infty$ for some constant $a_{1} \geq 0$. By Lemma 2.1. the sequence $\left\{r_{i}\right\}_{i=1}^{\infty}$ has a subsequence which we may assume without loss of generality to be the sequence itself such that $w\left(r_{i}\right) \rightarrow a_{2}$ as $i \rightarrow \infty$ where $a_{2}=0, w_{\infty}$, or $w_{1}$ with $w_{1}$ being given by (2.3). By (2.5), Lemma 2.5. Lemma 2.6, and the l'Hospital rule,

$$
\begin{aligned}
\frac{(n-1)}{m} a_{1} & =\frac{(n-1)}{m} \lim _{i \rightarrow \infty} r_{i}^{n-2} v\left(r_{i}\right)^{m} \\
& =\beta \lim _{i \rightarrow \infty} \frac{\int_{r_{i}}^{\infty} s v(s) d s}{r_{i}^{2-n}}+\lim _{i \rightarrow \infty} \frac{\int_{r_{i}}^{\infty} \frac{\alpha-n \beta}{s^{n-1}}\left(\int_{0}^{s} z^{n-1} v(z) d z\right) d s}{r_{i}^{2-n}} \\
& =\frac{\beta}{n-2} \lim _{i \rightarrow \infty} r_{i}^{n} v\left(r_{i}\right)+\frac{\alpha-n \beta}{n-2} \lim _{i \rightarrow \infty} \int_{0}^{r_{i}} z^{n-1} v(z) d z \\
& =\frac{\beta}{n-2} \lim _{i \rightarrow \infty} r_{i}^{n-2} v\left(r_{i}\right)^{m} \cdot \lim _{i \rightarrow \infty} r_{i}^{2} v\left(r_{i}\right)^{1-m}+\frac{\alpha-n \beta}{n-2} \int_{0}^{\infty} z^{n-1} v(z) d z \\
& =\frac{\beta}{n-2} a_{1} a_{2}+\frac{\alpha-n \beta}{n-2} \int_{0}^{\infty} z^{n-1} v(z) d z .
\end{aligned}
$$

Hence,

$$
\frac{\alpha-n \beta}{a_{1}} \int_{0}^{\infty} z^{n-1} v(z) d z=\frac{(n-1)(n-2)}{m}-\beta a_{2} .
$$

By (2.4) and (3.4),

$$
\begin{aligned}
-(n-1) \lim _{i \rightarrow \infty} \frac{r_{i} v^{\prime}\left(r_{i}\right)}{v\left(r_{i}\right)} & =\beta \lim _{i \rightarrow \infty} r_{i}^{2} v\left(r_{i}\right)^{1-m}+\lim _{i \rightarrow \infty} \frac{(\alpha-n \beta)}{r_{i}^{n-2} v\left(r_{i}\right)^{m}} \int_{0}^{r_{i}} z^{n-1} v(z) d z \\
& =\frac{(n-1)(n-2)}{m} .
\end{aligned}
$$


Hence,

$$
\lim _{i \rightarrow \infty} \frac{r_{i} v^{\prime}\left(r_{i}\right)}{v\left(r_{i}\right)}=-\frac{(n-2)}{m}
$$

By (1.2), (1.3) and (1.9),

$$
\frac{m \alpha}{\beta}<n-2
$$

holds. Hence, there exists a constant $\varepsilon>0$ such that

$$
\frac{m \alpha}{\beta}<n-2-\varepsilon \text {. }
$$

By (3.7) and Lemma 3.1 (3.1) and (3.2) hold. Then by (3.1), (3.2) and (3.7),

$$
\begin{aligned}
& 0>\frac{r v^{\prime}(r)}{v(r)}>-\frac{\alpha}{\beta}>-\frac{n-2}{m}+\frac{\varepsilon}{m} \quad \forall r>0 \\
\Rightarrow & \lim _{i \rightarrow \infty} \frac{r_{i} v^{\prime}\left(r_{i}\right)}{v\left(r_{i}\right)} \geq-\frac{n-2}{m}+\frac{\varepsilon}{m},
\end{aligned}
$$

which contradicts (3.6). Hence, no such sequence $\left\{r_{i}\right\}_{i=1}^{\infty}$ exists, and the lemma follows.

Lemma 3.3. Let $\rho>0, m, n, \alpha>n \beta$ satisfy (1.2), (1.3) and (1.9). Then there exists a constant $\varepsilon \in\left(0, \min \left(1, w_{\infty} / 2\right)\right)$ such that for any $R_{0}>1$ there exists $r^{\prime}>R_{0}$ such that

$$
w\left(r^{\prime}\right) \geq \varepsilon
$$

Proof. Suppose the lemma is false. Then

$$
\lim _{r \rightarrow \infty} w(r)=0 .
$$

We claim that

$$
\lim _{r \rightarrow \infty} \frac{r v^{\prime}(r)}{v(r)}=0 .
$$

By the proof of Lemma 3.2 there exists a constant $\varepsilon>0$ such that (3.8) holds. Suppose (3.10) does not hold. Then by (3.8) and (3.9) there exists a sequence $\left\{r_{i}\right\}_{i=1}^{\infty}, r_{i} \rightarrow \infty$ as $i \rightarrow \infty$, such that $r_{i} v^{\prime}\left(r_{i}\right) / v\left(r_{i}\right) \rightarrow a_{3}$ as $i \rightarrow \infty$ for some constant $a_{3}$ satisfying

$$
-\frac{n-2}{m}+\frac{\varepsilon}{m} \leq a_{3}<0
$$

and (3.5) holds. By Lemma 3.2, (3.5), (3.9) and (3.11), we get

$$
-(n-1) \lim _{i \rightarrow \infty} \frac{r_{i} v^{\prime}\left(r_{i}\right)}{v\left(r_{i}\right)}=0 \quad \text { if } v \in L^{1}\left(\mathbb{R}^{n}\right),
$$

and if $v \notin L^{1}\left(\mathbb{R}^{n}\right)$, then by the l'Hospital rule,

$$
\begin{aligned}
-(n-1) \lim _{i \rightarrow \infty} \frac{r_{i} v^{\prime}\left(r_{i}\right)}{v\left(r_{i}\right)} & =(\alpha-n \beta) \lim _{i \rightarrow \infty} \frac{r_{i}^{n-1} v\left(r_{i}\right)}{(n-2) r_{i}^{n-3} v\left(r_{i}\right)^{m}+m r_{i}^{n-2} v\left(r_{i}\right)^{m-1} v^{\prime}\left(r_{i}\right)} \\
& =(\alpha-n \beta) \lim _{i \rightarrow \infty} \frac{r_{i}^{2} v\left(r_{i}\right)^{1-m}}{n-2+m\left(r_{i} v^{\prime}\left(r_{i}\right) / v\left(r_{i}\right)\right)} \\
& =\frac{\alpha-n \beta}{n-2+m a_{3}} \cdot \lim _{i \rightarrow \infty} r_{i}^{2} v\left(r_{i}\right)^{1-m} \\
& =0 .
\end{aligned}
$$


Hence,

$$
a_{3}=\lim _{i \rightarrow \infty} \frac{r_{i} v^{\prime}\left(r_{i}\right)}{v\left(r_{i}\right)}=0,
$$

which contradicts (3.11). Thus, no such sequence $\left\{r_{i}\right\}_{i=1}^{\infty}$ exists and (3.10) follows. Since

$$
w^{\prime}(r)=\frac{2 w(r)}{r}\left(1+\frac{1-m}{2} \cdot \frac{r v^{\prime}(r)}{v(r)}\right)
$$

by (3.10) there exists a constant $R_{0}>0$ such that

$$
w^{\prime}(r)>0 \quad \forall r \geq R_{0},
$$

which contradicts (3.9) and the lemma follows.

We are now ready for the proof of Theorem 1.1.

Proof of Theorem 1.1. We divide the proof into two cases.

Case 1: $\alpha \leq n \beta$. By Corollary 2.3, Lemma 2.4 and Lemma 2.6, we get (1.10).

Case 2: $\alpha>n \beta$. By Lemma 2.5 there exists a constant $C_{1}>0$ such that (2.1) holds. Let $0<\varepsilon<\min \left(1, w_{\infty} / 2\right)$ be as in Lemma 3.3. Suppose there exists a sequence $\left\{r_{i}\right\}_{i=1}^{\infty}, r_{i} \rightarrow \infty$ as $i \rightarrow \infty$, such that $w\left(r_{i}\right)<\varepsilon$ for all $i \in \mathbb{Z}^{+}$. Then by Lemma 3.3 there exists a subsequence of $\left\{r_{i}\right\}_{i=1}^{\infty}$ which we may assume without loss of generality to be the sequence itself and a sequence $\left\{r_{i}^{\prime}\right\}_{i=1}^{\infty}$ such that $r_{i}<r_{i}^{\prime}<r_{i+1}$ for all $i=1,2, \ldots$ and

$$
w\left(r_{i}\right)<\varepsilon<w\left(r_{i}^{\prime}\right) \quad \forall i=1,2, \ldots
$$

By (3.12) and the intermediate value theorem, for any $i=1,2, \ldots$, there exists $a_{i} \in\left(r_{i}, r_{i}^{\prime}\right)$ such that

$$
w\left(a_{i}\right)=\varepsilon \quad \forall i=1,2, \ldots
$$

Hence, $a_{i} \rightarrow \infty$ as $i \rightarrow \infty$ and

$$
\lim _{i \rightarrow \infty} w\left(a_{i}\right)=\varepsilon
$$

This contradicts Lemma 2.1 and Remark 2.2. Hence no such sequence $\left\{r_{i}\right\}_{i=1}^{\infty}$ exists. Thus there exists a constant $R_{1}>1$ such that $w(r) \geq \varepsilon$ for all $r \geq R_{1}$. Hence (2.10) holds with $C_{2}=\min \left(\varepsilon, \min _{1 \leq r \leq R_{1}} w(r)\right)>0$. By Corollary 2.3 we get (1.10) and the theorem follows.

Proof of Corollary 1.3. By Theorem 1.1.

$$
\begin{aligned}
|x|^{2} v_{\lambda}(x)^{1-m} & =(\lambda|x|)^{2} v(\lambda x)^{1-m} \\
& \rightarrow \frac{2(n-1)(n(1-m)-2)}{(1-m)(\alpha(1-m)-2 \beta)} \quad \text { uniformly on } \mathbb{R}^{n} \backslash B_{R}(0)
\end{aligned}
$$

as $\lambda \rightarrow \infty$ for any $R>0$ and the corollary follows. 


\section{REFERENCES}

[CSZ] Huai-Dong Cao, Xiaofeng Sun, and Yingying Zhang, On the structure of gradient Yamabe solitons, Math. Res. Lett. 19 (2012), no. 4, 767-774. MR3008413

[CMM] Giovanni Catino, Carlo Mantegazza, and Lorenzo Mazzieri, On the global structure of conformal gradient solitons with nonnegative Ricci tensor, Commun. Contemp. Math. 14 (2012), no. 6, 1250045, 12. MR2989649

[DS1] Panagiota Daskalopoulos and Natasa Sesum, On the extinction profile of solutions to fast diffusion, J. Reine Angew. Math. 622 (2008), 95-119, DOI 10.1515/CRELLE.2008.066. MR2433613 (2009e:35146)

[DS2] Panagiota Daskalopoulos and Natasa Sesum, The classification of locally conformally flat Yamabe solitons, Adv. Math. 240 (2013), 346-369, DOI 10.1016/j.aim.2013.03.011. MR3046313

[GP] B. H. Gilding and L. A. Peletier, On a class of similarity solutions of the porous media equation, J. Math. Anal. Appl. 55 (1976), no. 2, 351-364. MR0436751 (55 \#9691a)

[H1] Shu-Yu Hsu, Singular limit and exact decay rate of a nonlinear elliptic equation, Nonlinear Anal. 75 (2012), no. 7, 3443-3455, DOI 10.1016/j.na.2012.01.009. MR.2891179

[H2] S.Y. Hsu, Some properties of the Yamabe soliton and the related nonlinear elliptic equation, Calc. Var. Partial Differential Equations 49 (2014), no. 1-2, 307-321. MR3148118

[PZ] M. A. Peletier and Hong Fei Zhang, Self-similar solutions of a fast diffusion equation that do not conserve mass, Differential Integral Equations 8 (1995), no. 8, 2045-2064. MR1348964 (96f:35080)

[V1] Juan Luis Vázquez, Smoothing and decay estimates for nonlinear diffusion equations. Equations of porous medium type. Oxford Lecture Series in Mathematics and its Applications, vol. 33, Oxford University Press, Oxford, 2006. MR 2282669 (2007k:35008)

[V2] Juan Luis Vázquez, The porous medium equation. Mathematical theory. Oxford Mathematical Monographs, The Clarendon Press, Oxford University Press, Oxford, 2007. MR2286292 (2008e:35003)

Department of Mathematics, National Chung Cheng University, 168 University Road, Min-Hsiung, Chia-Yi 621, Taiwan, Republic of China

E-mail address: syhsu@math.ccu.edu.tw 